\title{
Mucosa-associated lymphoid tissue lymphoma of the parotid gland - a case report
}

\author{
Delia Muntean', Sorin Dudea², Manuela Lenghel², Carolina Solomon², Teodora Iuga ${ }^{3}$, \\ Horațiu Rotar 4
}

${ }^{1}$ Emergency County Hospital Cluj-Napoca, Radiology Department, ${ }^{2 " I u l i u ~ H a t ̧ i e g a n u ” ~ U n i v e r s i t y ~ o f ~ M e d i c i n e ~ a n d ~}$ Pharmacy, Radiology Department, ${ }^{3}$ Emergency County Hospital Cluj-Napoca, Maxillofacial Surgery and Implantology Department, "“Iuliu Hațieganu" University of Medicine and Pharmacy, Maxillofacial Surgery and Implantology Department

\begin{abstract}
Mucosa-associated lymphoid tissue (MALT) lymphoma of the parotid gland is a rare entity among head and neck tumours. We report a case of a patient with a medical history of diffuse large B-cell Non-Hodgkin Lymphoma with complete remission following chemotherapy, who presented seven years afterwards with a progressive painless hard swelling of the right parotid gland. Ultrasonography followed by contrast-enhanced MRI features was consistent with a malignant tumour. Consequently, fine and core needle aspiration biopsy were performed under ultrasound guidance and the final histopathological result was MALT lymphoma of the parotid gland. The most important particularity of the case is the sequential development of two different histopathological types of Non-Hodgkin Lymphoma, both of B-cell lineage.
\end{abstract}

Keywords: parotid; mucosa-associated lymphoid tissue lymphoma; ultrasound; MRI

\section{Introduction}

Primary salivary gland lymphoma is uncommon, accounting for $1.7-3.1 \%$ of all salivary gland malignancies [1]. Lymphomas are generally classified into Hodgkin and Non-Hodgkin Lymphoma (NHL) and can be further subdivided into intranodal or extranodal types. Mucosaassociated lymphoid tissue (MALT) lymphoma represents a low-grade marginal zone B-cell NHL affecting various extranodal sites, such as gastric mucosa, lacrimal and salivary glands $[2,3]$.

\section{Case report}

We report a case of a 71-year-old woman referred to the Oromaxillofacial Surgery Department for facial

Received 13.12.2020 Accepted 14.03.2021

Med Ultrason

2021, Vol. 23, No 3, 364-366

Corresponding author: Manuela Lenghel, $\mathrm{MD}, \mathrm{PhD}$

Radiology Department, "Iuliu Hațieganu"

University of Medicine and Pharmacy

3-5 Clinicilor Str., 400006, Cluj-Napoca, Romania

Phone: +40740.423 .025$

E-mail: lenghel.manuela@gmail.com asymmetry and a progressive painless hard swelling of the right parotid gland, first noticed three months before admission. In 2013, the patient was previously treated for diffuse large B-cell NHL (DLBCL), Ann Arbor stage III with cervical, axillary, and retroperitoneal lymph nodes involvement. The patient underwent chemotherapy with rituximab, cyclophosphamide, doxorubicin, vincristine, and prednisone (R-CHOP) and achieved complete response assessed on the annual imaging follow-up.

The first-line performed imaging method was ultrasonography (US) which revealed a hypoechoic, heterogeneous, irregular mass with anarchic vascularity on colour Doppler US and soft appearance with small stiff areas at real-time elastography (fig 1). No calcifications or necrotic areas were encountered. Based on the US appearance and the patient's clinical history, a malignant lesion was suspected, with a high possibility of a lymphoma variant.

Contrast-enhanced MRI features were also consistent with malignancy. MRI demonstrated an ill-defined mass within the superficial lobe of the right parotid gland, presenting marked diffusion restriction (ADC $0.588 \mathrm{x}$ $10^{-3} \mathrm{~mm}^{2} / \mathrm{s}$ ) and increased homogenous enhancement (fig 2). Fine-needle aspiration biopsy was performed and 


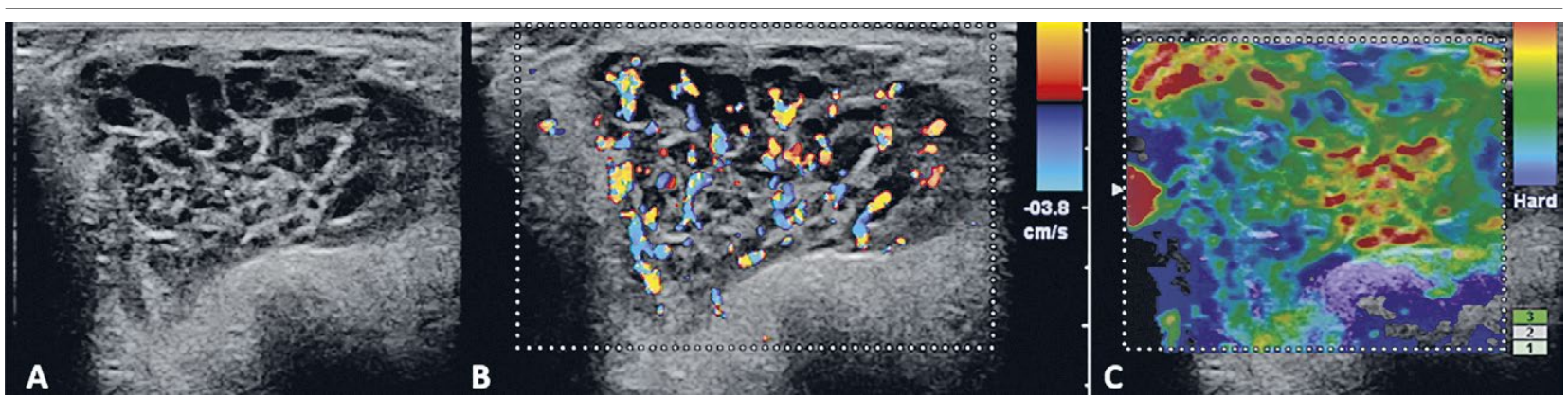

Fig 1. A. Ultrasonography of the right parotid gland shows a hypoechoic, heterogeneous lesion, with irregular and ill-defined margins. B. Color Doppler-US reveals an anarchic distribution of the vessels. C. Real-time elastography depicts a soft appearance with small stiff areas within the parotid mass.

the cytologic exam revealed a hypercellular smear with matured transformed lymphocytes, suggestive of lymphoma.

For a general assessment, the patient underwent a cervical and thoraco-abdominopelvic contrast-enhanced CT scan which showed no abnormalities apart from the spontaneously hyperdense parotid mass with homogeneous enhancement (fig 3).

Consequently, core needle biopsy was performed(fig 4) and the definitive histopathological result was MALT lymphoma, confirmed immunohistochemically (positive CD20, BCL2). The patient started R-CHOP chemotherapy, presenting a favourable outcome and total resolution of the lymphoma at 6 months imaging control performed with PET-CT.

\section{Discussion}

The case's most important particularity represents the appearance of the second type of lymphoid proliferation, located in the parotid gland (MALT lymphoma), in a patient with a clinical history of DLBCL. MALT lymphoma usually presents an indolent clinical course [4]. In the presented case, however, a progressive increase of the parotid region was noticed over three months before admission.

Normally, the salivary tissue does not contain any lymphocytes, thus states of chronic inflammation are responsible for lymphoid proliferation and the development of MALT lymphoma [5]. An increased incidence of parotid lymphoma was reported in patients with underlying autoimmune conditions (Sjögren's Syndrome) or chronic infections [6,7]. Our patient's medical history was, however, negative for the above-mentioned pathologies.

US is frequently used as the first imaging technique for the evaluation of the salivary glands, allowing the real-time assessment of parotid lesions with an excellent spatial resolution [8]. In our case, US depicted the malig- nant features of the lesion and played an essential role in biopsy guidance.

Cross-sectional imaging with $\mathrm{CT}$ and, most important, multiparametric MRI, is performed to obtain com-

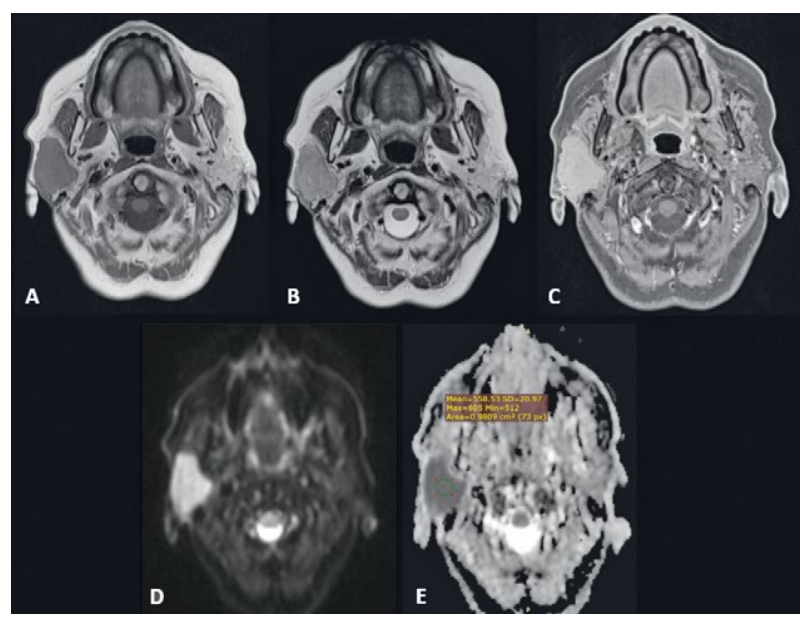

Fig 2. MRI reveals a mass within the superficial lobe of the right parotid gland with low-intensity signal on T1 (A) and T2 (B) weighted imaging compared to the parotid parenchyma, with increased homogeneous contrast uptake (C) and increased restricted diffusion with low $\mathrm{ADC}$ values $(\mathrm{D}, \mathrm{E})$.

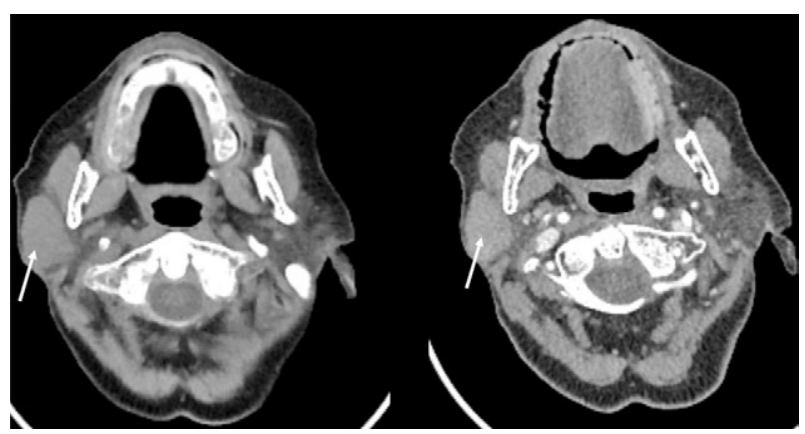

Fig 3. A. Native CT reveals a spontaneously hyperdense solid mass occupying almost the entire right parotid gland. B. Contrast-enhanced CT shows diffuse homogeneous enhancement of the lesion. 


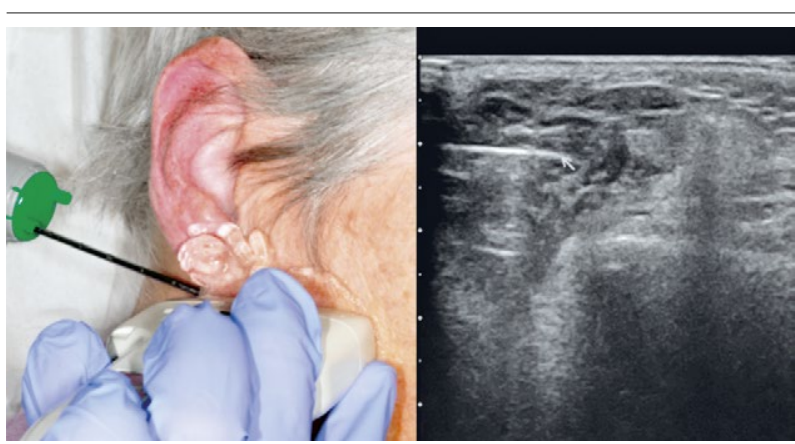

Fig 4. Core biopsy of the parotid mass optimally performed under US guidance.

plementary information about the lesion's structure, offering a complete map of local extension in malignancies, including perineural spread or bone invasion [9].

MALT lymphoma of the parotid gland can present various imaging aspects, the most suggestive proving to be the solitary or diffuse solid-cystic appearance [10]. On MRI, head-neck lymphomas generally present important restricted diffusion due to the high cellularity of the lymphoid tissue [11], as in our case report. However, there is no single pathognomonic imaging feature for MALT lymphoma and there is significant overlap with several other parotid masses, thus producing diagnostic pitfalls. The differential diagnosis includes both benign entities such as Warthin tumour, lymphoepithelial lesions, and other malignancies such as other types of lymphoma, adenoid cystic carcinoma or mucoepidermoid carcinoma $[3,10]$.

Our patient sequentially developed two different types of NHL, both of B-cell lineage. A correlation between MALT lymphoma and DLBCL might represent the presence of aberrant somatic hypermutation, a genetic instability mechanism proved to occur in both disorders [12]. Chemotherapy in long-term surviving patients also produces changes in the phenotype of malignant cells [13]. Therefore, patients diagnosed with one type of NHL, even after achieving a complete response, should be closely followed-up to detect relapses of other NHL types [14]

In conclusion, although rare, parotid lymphoma should always be included in the differential diagnosis of parotid masses, especially in a patient with previously diagnosed lymphoid neoplasia. Ultrasonography is the first-line imaging method to assess parotid glands, confirming the presence of a lesion and playing an essential role in biopsy guidance. MRI offers additional structural details of the parotid parenchyma and draws a complete map of the lesion extent. Ultimately, the histopathologi- cal exam and immunohistochemical staining remain the gold standard methods for the final diagnosis of parotid lymphoma types.

\section{References}

1. Jamal B. Treatment of Parotid Non-Hodgkin Lymphoma: A Meta-Analysis. J Glob Oncol 2018;4:1-6.

2. Zhang XY, Wang ZM. Relevance on the diagnosis of malignant lymphoma of the salivary gland. World J Clin Cases 2020;8:2717-2726.

3. Aydın S, Demir MG, Barısık NÖ. Extranodal marginal zone lymphoma of the parotid gland. J Maxillofac Oral Surg 2016;15(Suppl 2):346-350.

4. Anacak Y, Miller RC, Constantinou N, et al. Primary mucosa-associated lymphoid tissue lymphoma of the salivary glands: a multicenter Rare Cancer Network study. Int J Radiat Oncol Biol Phys 2012;82:315-320.

5. Ren Y, Huang L, Han Y, et al. 18F-FDG PET/CT for staging and response assessment of primary parotid MALT lymphoma with multiple sites involvement: A case report. Medicine (Baltimore) 2019;98:e14270.

6. Zucca E, Bertoni F. The spectrum of MALT lymphoma at different sites: biological and therapeutic relevance. Blood 2016;127:2082-2092.

7. Bădărînză M, Serban O, Maghear L, et al. Shear wave elastography as a new method to identify parotid lymphoma in primary Sjögren Syndrome patients: an observational study. Rheumatol Int 2020;40:1275-1281.

8. Bhatia KSS, Dai YL. Routine and Advanced Ultrasound of Major Salivary Glands. Neuroimaging Clin N Am 2018;28:273-293.

9. Abdel Razek AAK, Mukherji SK. State-of-the-Art Imaging of Salivary Gland Tumors. Neuroimaging Clin N Am 2018;28:303-317.

10. Zhu L, Wang P, Yang J, Yu Q. Non-Hodgkin lymphoma involving the parotid gland: $\mathrm{CT}$ and MR imaging findings. Dentomaxillofac Radiol 2013;42:20130046.

11. Kato H, Kanematsu M, Goto H, et al. Mucosa-associated lymphoid tissue lymphoma of the salivary glands: MR imaging findings including diffusion-weighted imaging. Eur J Radiol 2012;81:e612-e617.

12. Deutsch AJ, Aigelsreiter A, Staber PB, et al. MALT lymphoma and extranodal diffuse large B-cell lymphoma are targeted by aberrant somatic hypermutation. Blood 2007;109:3500-3504.

13. Matsuo T, Ichimura K, Shinagawa K. Orbital MALT lymphoma, abdominal hodgkin lymphoma, and systemic diffuse large B-cell lymphoma develop sequentially in one patient. J Clin Exp Hematop 2012;52:41-49.

14. Yang X, Min X, He W. Sequential development of multifocal recurrent non-Hodgkin's lymphoma of mucosa-associated lymphoid tissue and diffuse large B-Cell lymphoma in a single patient: A case report. Medicine (Baltimore) 2018;97:e10845. 